\title{
Route optimization security in mobile IPv6 wireless networks: a Test-bed experience
}

\begin{abstract}
Route Optimization (RO) is standard in Mobile IPv6 (MIPv6) to route packets between Mobile Node $(\mathrm{MN})$ and Correspondent Node $(\mathrm{CN})$ using shortest possible path. It provides better bandwidth and faster transmission. RO greatly increases the security risk. In this paper, focus is given on enhanced security scheme in terms of RO based Test-bed evaluation experiment. An enhanced security algorithm is developed on top of MIPv6 RO to secure data. This algorithm is able to detect and prevent the attacker from modifying the data with using an encryption algorithm by cost of little bit increase but tolerable delay. The real-time network Test-bed is implemented to prove the efficiency of proposed method. The experimental results show that the proposed security scheme increases the security performance of the network. This gives advantage of safe communication that can significantly improve the data security of RO while maintaining the quality of other network performance.
\end{abstract}

Keyword: Mobile IPv6; Route Optimization; Security; IPv6 Test-bed 